Quim. Nova, Vol. 34, No. 7, 1182-1187, 2011

\title{
CONSTITUINTES QUÍMICOS E ATIVIDADE LEISHMANICIDA DE Gustavia elliptica (LECYTHIDACEAE)
}

\author{
Maria de Fátima Oliveira Almeida, Ana Cláudia Rodrigues de Melo, Maria Lúcia Belém Pinheiro, Jefferson Rocha de \\ Andrade Silva e Afonso Duarte Leão de Souza* \\ Departamento de Química, Universidade Federal do Amazonas, Av. General Rodrigo Octávio Jordão Ramos, 3000, Campus \\ Universitário, setor Sul, 69077-000 Manaus - AM, Brasil \\ Andersson Barison e Francinete Ramos Campos \\ Departamento de Química, Universidade Federal do Paraná, Centro Politécnico, CP 19081, 81531-990 Curitiba - PR, Brasil \\ Ana Cláudia Fernandes Amaral \\ Instituto de Tecnologia de Fármacos, Farmanguinhos, Fundação Oswaldo Cruz, Av. Brasil, 4365, 21041-250, Rio de Janeiro - RJ, \\ Brasil \\ Gerzia Maria de Carvalho Machado e Leonor Laura Pinto Leon \\ Departamento de Imunologia, Fundação Oswaldo Cruz, Av. Brasil, 4365, 21045-900 Rio de Janeiro - RJ, Brasil
}

Recebido em 21/10/10; aceito em 17/2/11; publicado na web em 15/4/11

\begin{abstract}
CHEMICAL CONSTITUENTS AND LEISHMANICIDAL ACTIVITY OF Gustavia elliptica (LECYTHIDACEAE). The phytochemical investigation of the stem bark and leaves of G. elliptica provided a mixture of the norisoprenoids blumenol B and 6-epiblumenol B along with the triterpenes friedelin, as the major constituent, friedelanol, ursa- $9(11), 12$-dien-3-ol, $\alpha$-amyrin, $\beta$-amyrin, morentenol, epifriedelanol, as well as the sesquiterpenes trans-caryophyllene, $\alpha$-humulene, ethyl hydnocarpate and other fatty acid esters. The identification of the compounds was performed on basis of spectrometric methods such as GC-MS, IR, MS and 1D and 2D NMR. Stem bark extracts showed significant leishmanicidal activity against promastigote forms of Leishmania braziliensis, with the best results for the chloroform extract.
\end{abstract}

Keywords: Gustavia elliptica; blumenol B; 6-epiblumenol B.

\section{INTRODUÇÃO}

O gênero Gustavia, pertencente à família Lecythidaceae, possui cerca de 40 espécies distribuídas na América Tropical desde a Costa Rica até o extremo sul da Amazônia, estando também presente no nordeste brasileiro. ${ }^{1}$ A principal atividade relatada na etnomedicina é a utilização dos frutos, cascas ou folhas jovens, em forma de emplastros, pelos índios da tribo Palikur na Guiana Francesa contra leishmaniose. ${ }^{2}$ Poucos são os estudos fitoquímicos realizados com espécies do gênero Gustavia, os quais descrevem, principalmente, a presença de ácidos graxos, triterpenos e esteroides. ${ }^{3-6}$ Entretanto, ensaios biológicos realizados com extratos e frações de G. augusta revelaram significante atividade anti-inflamatória, enquanto que o estudo químico desta espécie resultou no isolamento de ácido betulínico, um dos mais potentes inibidores de células tumorais. ${ }^{7}$ A partir de $G$. hexapetala foi isolado um novo inibidor de células cancerígenas humanas, o depsídeo gustastatina, juntamente com o ácido betulínico. ${ }^{4,5}$

A espécie Gustavia elliptica S. A. Mori é restrita às terras firmes das florestas da Amazônia Central, com grande ocorrência nas vizinhanças da cidade de Manaus, onde suas árvores, conhecidas como ripeiro, general, castanharana ou mucurão, chegam a atingir cerca de $22 \mathrm{~m}$ de altura e $35 \mathrm{~cm}$ de diâmetro. ${ }^{1,8}$ Como as demais espécies do gênero, sua madeira apresenta um odor fétido, atribuído à emanação de dissulfeto de etila, bem como de outros compostos voláteis de enxofre, que lhe conferem parcial proteção contra alguns tipos de insetos, especialmente besouros. ${ }^{5}$

\footnotetext{
*e-mail: souzadq@ufam.edu.br
}

Na busca contínua por compostos com atividade leishmanicida de plantas da Amazônia, foi realizada a investigação fitoquímica da casca do caule e das folhas de Gustavia elliptica, resultando na obtenção do norisoprenoide- $\mathrm{C}_{13}$ blumenol $\mathrm{B}$ e de seu epímero 6-epiblumenol B, ésteres de ácidos graxos, sesquiterpenos e triterpenos, entre os quais a friedelina encontrada em quantidade majoritária em todos os extratos analisados. Em adição, extratos, frações e compostos de G. elliptica foram ensaiados in vitro contra formas promastigotas infectantes de Leishmania braziliensis e L. guyanensis.

\section{PARTE EXPERIMENTAL}

\section{Procedimentos gerais}

Os espectros de infravermelho foram obtidos em pastilhas de $\mathrm{KBr}$ em um espectrômetro Perkin Elmer Spectrum 2000. As medidas de ponto de fusão foram obtidas em um aparelho Metler Toledo FP 62, com taxa de aquecimento de $5{ }^{\circ} \mathrm{C} \mathrm{min}-1$ e não foram corrigidas. Os experimentos de RMN 1D $\left({ }^{1} \mathrm{H},{ }^{13} \mathrm{C}\left\{{ }^{1} \mathrm{H}\right\}\right.$ e $\left.1 \mathrm{D}-\mathrm{NOE}\right)$ e $2 \mathrm{D}$ (COSY, $\mathrm{HSQC}, \mathrm{HMBC}$ e $J$-Resolvido) foram adquiridos em $\mathrm{CDCl}_{3}$ a 295 $\mathrm{K}$ em espectrômetros de RMN Varian Inova 500 ou Bruker Avance 400, cujos magnetos de 11,7 Tesla, para o primeiro, e 9,4 Tesla, para o segundo, permitiram observar os núcleos de ${ }^{1} \mathrm{H}$ a 500 e $400 \mathrm{MHz}$ e os ${ }^{13} \mathrm{C}$ a 125 e $100 \mathrm{MHz}$, respectivamente. Os experimentos de correlação direta ${ }^{1} \mathrm{H}-{ }^{13} \mathrm{C}$ (HSQC) e a longa distância (HMBC) foram otimizados para constantes de acoplamento ${ }^{1} J_{(\mathrm{C}, \mathrm{H})}, 140 \mathrm{~Hz}, \mathrm{e}^{\mathrm{LD}} J_{(\mathrm{C}, \mathrm{H})}$, $8 \mathrm{~Hz}$, respectivamente. Os deslocamentos químicos de RMN de ${ }^{1} \mathrm{H}$ e ${ }^{13} \mathrm{C}$ são expressos em ppm $(\delta)$ em relação ao sinal do TMS em 0,00 ppm, como referência interna, e as constantes de acoplamento 
$(J)$ em Hz. As separações por cromatografia em coluna (CC) foram realizadas com sílica gel 60G (0,025-0,063 mm, Merck), enquanto que as análises por cromatografia em camada delgada (CCD) foram realizadas em cromatoplacas pré-fabricadas de sílica gel $60 \mathrm{~F}_{254}$ (Merck) com 0,2 mm de espessura. As placas cromatográficas foram reveladas por borrifação com solução etanólica de ácido sulfúrico $5 \%$ ou vanilina sulfúrica $5 \%$, seguida por aquecimento a $110{ }^{\circ} \mathrm{C}$, reagente de Liberman Buchard, solução de NP/PEG e exposição à luz ultravioleta em 254 e $366 \mathrm{~nm}$.

As análises por CG-EM dos constituintes das folhas foram realizadas em um cromatógrafo a gás acoplado a um espectrômetro de massas Shimadzu QP2010, equipado com injetor split/splitless e uma coluna capilar de sílica fundida Agilent DB-5 de baixa polaridade (5\% metilsilicone e $95 \%$ fenil), de $30 \mathrm{~m}$ de comprimento, $0,25 \mathrm{~mm}$ de d.i. e filme de 0,25 $\mu \mathrm{m}$ de espessura. Condições das análises cromatográficas: injetor a $250{ }^{\circ} \mathrm{C}$; forno: $250-290{ }^{\circ} \mathrm{C}$ a 10 ${ }^{\circ} \mathrm{C} \min ^{-1}, 290{ }^{\circ} \mathrm{C}$ por $30 \mathrm{~min}$; hélio como gás de arraste a um fluxo de 2,0 $\mathrm{mL} \mathrm{min}^{-1}$; faixa de massas de 20 a 500 Daltons; energia de ionização (IE) de 70 eV.

As análises por CG-EM dos constituintes da casca do caule foram realizadas em um cromatógrafo a gás acoplado a um espectrômetro de massas Agilent Techologies $6896 \mathrm{~N}$, equipado com injetor split/ splitless e com uma coluna capilar de sílica fundida HP-5MS (5\% difenil e $95 \%$ dimetilpolissiloxano) de $30 \mathrm{~m}$ de comprimento, 0,25 $\mathrm{mm}$ de d.i. e filme de $0,25 \mu \mathrm{m}$ de espessura. Condições das análises cromatográficas: injetor a $250{ }^{\circ} \mathrm{C}$; forno: $200-305^{\circ} \mathrm{C}$ a $10^{\circ} \mathrm{C} \mathrm{min}{ }^{-1}$, $305^{\circ} \mathrm{C}$ por $35 \mathrm{~min}$; hélio como gás de arraste a um fluxo de $2,0 \mathrm{~mL}$ $\min ^{-1}$; faixa de massas de 30 a 500 Daltons; energia de ionização (IE) de $70 \mathrm{eV}$. Os constituintes foram identificados pela análise das fragmentações observadas em seus respectivos espectros de massas e por comparações com as bibliotecas de massas Wiley 7n e Nist 98 e com dados descritos na literatura. ${ }^{9}$ Alguns ainda tiveram seus tempos de retenção comparados com os de padrões obtidos e identificados por membros da equipe de autores.

\section{Material vegetal}

Cascas do caule e folhas de Gustavia elliptica foram coletadas em novembro de 2001 e fevereiro de 2006, respectivamente, na fazenda experimental da Universidade Federal do Amazonas, no km 38 da BR-174, e identificadas pelo pesquisador M. Hopkins, do Instituto Nacional de Pesquisas da Amazônia (INPA). As exsicatas foram depositadas no herbário do Instituto de Ciências Biológicas da Universidade Federal do Amazonas (n 7280).

\section{Extração e isolamento dos constituintes químicos das folhas}

As folhas secas e trituradas (600 g) foram extraídas a frio em aparelho de ultrassom sucessivamente com diclorometano $(3 \times 2 \mathrm{~L})$ e metanol ( $3 \times 2 \mathrm{~L}$ ), por períodos de 20 min cada. Os extratos foram concentrados em evaporador rotativo à pressão reduzida, resultando nos extratos em diclorometano (10,5 g) e metanol (41,6 g), respectivamente. Parte do extrato em metanol $(12 \mathrm{~g})$ foi fracionada em CC com gel de sílica utilizando-se para eluição $\mathrm{CHCl}_{3}, \mathrm{CHCl}_{3} / \mathrm{MeOH}$ : (95:5), (9:1), (8:2), (7:3), (1:1) e (0:1). Da fração obtida na mistura de $\mathrm{CHCl}_{3} / \mathrm{MeOH}$ 95:5 (209,7 mg) foi feita nova CC de gel de sílica utilizando-se para eluição hexano/acetato de etila: (1:1), (4:6), (3:7), (8:2) e (0:1). Da fração 3:7 foi obtido um óleo viscoso de coloração levemente amarelada (7,9 mg), constituído pela mistura dos epímeros blumenol B e 6-epiblumenol B (1 e 2).

Parte do extrato em diclorometano $(9,9 \mathrm{~g})$ foi fracionada em uma CC de gel de sílica utilizando-se como eluente hexano/ acetato de etila em gradiente de polaridade crescente. A fração eluída em hexano apresentou uma grande quantidade de material floculoso de coloração branca, identificada como uma mistura de hidrocarbonetos $(\sim 0,23 \mathrm{~g})$. Da fração hexano/acetato de etila 9:1 $(1,98 \mathrm{~g})$ foi feita uma nova CC de gel de sílica com os mesmos solventes, porém com aumento de polaridade mais suave. Das frações eluídas com hexano/acetato de etila 99:1 e 98:2 foram obtidos, respectivamente os triterpenos friedelina (3) $(175,0 \mathrm{mg})$ e friedelanol (4) (168,0 g). Da fração obtida na proporção de 97:3 do eluente, foi feita nova CC de gel de sílica utilizando-se para eluição combinações de hexano/diclorometano/acetato de etila em várias proporções. Obteve-se nas frações 94:4:2 a 93:5:2 do eluente um sólido branco cristalino (47,0 mg), identificado como uma mistura de $\alpha$ e $\beta$-amirinas (5 e 6 ).

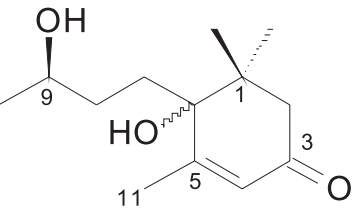

$16 \mathrm{~S} ; 2$ 6R

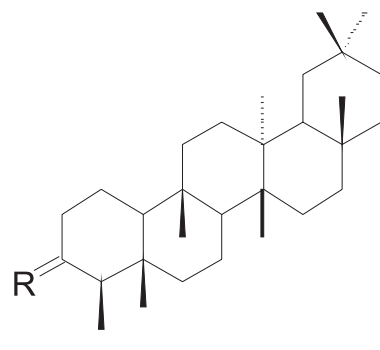

$3 \mathrm{R}=\mathrm{O} ; 4 \mathrm{R}=\beta-\mathrm{OH}, \mathrm{H}$

\section{Extração e isolamento dos constituintes químicos da casca do caule}

As cascas do caule, secas e moídas $(1,35 \mathrm{~kg})$, foram submetidas à extração exaustiva por maceração com etanol 95\%. Após eliminação do solvente em evaporador rotativo à pressão reduzida foi obtido um extrato bruto $(83,4 \mathrm{~g})$, parte do qual $(12,6 \mathrm{~g})$ foi particionada em clorofórmio e água (1:1), fornecendo os extratos em clorofórmio (6,5 g) e aquoso (2,8 g), além de uma fração insolúvel (2,8 g).

Uma parte do extrato em clorofórmio $(3,7 \mathrm{~g})$ foi submetida a uma nova partição com hexano e metanol 90\% (1:1), resultando nas sub-frações em hexano (1,5 g) e metanol (1,6 g). Outra parte desse extrato $(1,0 \mathrm{~g})$ foi fracionada em uma CC de gel de sílica utilizando-se para eluição hexano, diclorometano e metanol em gradiente de polaridade crescente. As frações resultantes foram comparadas em CCD, reunidas em três grupos (A-C) e analisadas por CG-EM, conforme as condições descritas nos procedimentos gerais. No grupo A $(45,4$ $\mathrm{mg}$ ), obtido por eluição com hexano, foi identificada uma mistura dos ésteres graxos palmitato, linoleato, oleato e estearato de etila (7-10). Do grupo B (30,0 mg), eluído com hexano/diclorometano (9:1), foi obtido o triterpeno friedelina (3) (30,0 mg) após sucessivas recristalizações em acetato de etila. Do grupo C (27,1 mg), proveniente da eluição com hexano/diclorometano (8:2), foi identificada uma mistura dos triterpenos 3 e friedelanol (4) (Tabela 1).

Parte da sub-fração em hexano $(1,0 \mathrm{~g})$ foi fracionada em sucessivas colunas de gel de sílica fazendo-se a eluição com misturas de hexano e diclorometano, em gradiente crescente de polaridade. As frações resultantes foram selecionadas e reunidas em seis grupos (D-I), eluídos com hexano/diclorometano 7:3 a 1:9, os quais foram submetidos a análises por CG-EM, conforme as condições descritas nos procedimentos gerais. No grupo D $(52,1 \mathrm{mg})$ foi identificada uma mistura de $\mathbf{3}, \mathbf{5}$ e $\mathbf{6}$. Ambos os grupos E (14,7 mg) e F (10,2 $\mathrm{mg}$ ) continham os triterpenos $\mathbf{5}$ e $\mathbf{6}$, moretenol (12) e ursa-9(11), 12dien-3-ol (13), tendo o grupo E também o epi-friedelanol (11). No Grupo G (25,3 mg) foram identificados 3, 7, 9, 10, o trans-cariofileno (14) e o hidnocarpato de etila (15). O grupo H (29,4 mg) continha apenas 3 e o grupo I (42,7 mg) estava constituído por 3, 11, 14 e o $\alpha$-humuleno (16) (Tabela 2). 
Tabela 1. Constituintes químicos identificados nas cascas do caule de Gustavia elliptica por CG-EM

\begin{tabular}{|c|c|c|c|c|}
\hline Extrato & Amostra & Constituintes & $\mathrm{TR} / \mathrm{min}$ & Abundância/\% \\
\hline \multirow[t]{7}{*}{ Clorofórmio } & Grupo A & palmitato de etila (7) & 25,43 & 42,84 \\
\hline & & linoleato de etila (8) & 30,36 & 14,96 \\
\hline & & oleato de etila (9) & 30,58 & 15,07 \\
\hline & & estearato de etila (10) & 31,41 & 15,87 \\
\hline & Grupo B & friedelina $(\mathbf{3})$ & 61,68 & 96,08 \\
\hline & Grupo C & friedelina $(\mathbf{3})$ & 61,21 & 95,18 \\
\hline & & friedelanol (4) & 61,72 & 4,82 \\
\hline \multirow[t]{19}{*}{ Hexano } & Grupo D & $\alpha$ - amirina $(\mathbf{5})$ & 59,12 & 6,42 \\
\hline & & $\beta$-amirina $(6)$ & 58,19 & 3,90 \\
\hline & & friedelina $(3)$ & 62,69 & 82,69 \\
\hline & Grupo E e F & epifriedelanol (11) & 61,17 & 4,77 \\
\hline & & $\alpha$ - amirina $(\mathbf{5})$ & 59,12 & $\sim 45,00$ \\
\hline & & $\beta$-amirina $(6)$ & 58,19 & $\sim 1,00$ \\
\hline & & Moretenol (12) & 60,67 & $\sim 1,00$ \\
\hline & & ursa-9(11), 12-dien-3-ol (13) & 57,48 & $\sim 1,00$ \\
\hline & Grupo G & trans-cariofileno (14) & 8,16 & 1,09 \\
\hline & & palmitato de etila (7) & 25,43 & 3,84 \\
\hline & & hidnocarpato de etila (15) & 27,04 & 27,78 \\
\hline & & oleato de etila (9) & 30,56 & 0,90 \\
\hline & & estearato de etila (10) & 31,41 & 0,44 \\
\hline & & friedelina $(\mathbf{3})$ & 61,66 & 48,26 \\
\hline & Grupo H & friedelina $(\mathbf{3})$ & 61,68 & 96,08 \\
\hline & Grupo I & trans-cariofileno (14) & 8,16 & 4,49 \\
\hline & & $\alpha$-humuleno (16) & 9,03 & 0,54 \\
\hline & & epifriedelanol (11) & 61,20 & 11,61 \\
\hline & & friedelina $(\mathbf{3})$ & 61,65 & 78,02 \\
\hline
\end{tabular}

Os constituintes foram identificados pela análise das fragmentações observadas em seus respectivos espectros de massas e por comparações com as bibliotecas de massas Wiley 7n e Nist 98 e com dados descritos na literatura. ${ }^{9}$ Alguns ainda tiveram seus tempos de retenção comparados com os de padrões obtidos e identificados por membros da equipe de autores.

\section{Ensaios biológicos}

Para os testes biológicos, foram utilizadas cepas de Leishmania (V) braziliensis (MHCAN/BR/98/R619), cedidas pela Dra. M. de F. Madeira, do Lab. de Protozologia, Depto. de Ciências Biológicas, ENSP/FIOCRUZ, e de L. (V) guyanensis (IM4216), cedidas pela Dra. A. M. R. Franco, do Lab. de Leishmaniose e Doença de Chagas, Instituto Nacional de Pesquisas da Amazônia (CPCS/INPA).

Formas promastigotas de $L$. (V) braziliensis e $L$. (V) guyanensis foram cultivadas a $26^{\circ} \mathrm{C} \mathrm{em}$ meio de cultura bifásico (sólido-líquido). Como fase sólida foi empregado o meio $\mathrm{NNN}^{10,11}$ constituído de ágar com $15 \%$ de sangue desfibrinado de coelho e como fase líquida meio Schneider's ${ }^{12}$ em pH 7,2 suplementado com $20 \%$ de soro fetal bovino (SFB) inativado e estéril e $2 \%$ de urina humana masculina esterilizada. Para análise, $10 \mathrm{mg}$ de cada amostra foram solubilizadas em $1 \mathrm{~mL}$ de DMSO, seguindo-se as diluições programadas. Foi medida a concentração inibitória de cada amostra para $50 \%$ das formas promastigotas $\left(\mathrm{CI}_{50} / 24 \mathrm{~h}\right)$, sendo que a faixa de concentração testada foi de 0,16 a $320 \mu \mathrm{g} \mathrm{mL} \mathrm{g}^{-1}$. Os valores de $\mathrm{CI}_{50} / 24 \mathrm{~h}$ foram determinados por meio de regressão linear. Todos os experimentos foram realizados em triplicata. Pentamidina foi usada como controle positivo. ${ }^{13} \mathrm{O}$ extrato em clorofórmio foi avaliado contra $L .(V)$ braziliensis e $L$. (V) guyanensis, enquanto que os extratos em hexano e metanol foram avaliados contra $L$. (V) braziliensis e o triterpeno friedelina (3) foi avaliado contra $L$. (V) guyanensis. Os resultados dos testes de atividade leishmanicida estão relacionados na Tabela 3.

\section{Friedelina (3)}

Sólido branco, cristais em agulhas, Tf $(250-253,6){ }^{\circ} \mathrm{C}$, não corrigido. EM (IE, $70 \mathrm{eV}), \mathrm{m} / \mathrm{z}(\%): 426[\mathrm{M}]^{+}(16), 273(20), 205(10) \mathrm{e}$ 69 (100). RMN de ${ }^{1} \mathrm{H}\left[500 \mathrm{MHz}, \mathrm{CDCl}_{3}, \delta, J(\mathrm{~Hz})\right]: 1,96(m ; \mathrm{H}-1 \mathrm{a})$, $1,68(d q ; J=13,0 ; 5,1 ; \mathrm{H}-1 \mathrm{~b}), 2,39(d d d ; J=13,7 ; 5,1 ; 1,9 ; \mathrm{H}-2 \mathrm{a})$, $2,29(m ; J=13,8 ; 13,6 ; 6,5 ; \mathrm{H}-2 \mathrm{~b}), 2,24(q ; J=6,5 ; \mathrm{H}-4), 1,75(m$; H-6a), 1,27 ( $m$; H-6b), 1,59 ( $m$; H-7a), 1,47 ( $m$; H-7b), 1,44 ( $m$; H-8a), 1,39 ( $m$; H-8b), 1,55 ( $m$; H-10), 1,38 ( $m$; H-11), 1,35 ( $m$; H-12), 1,47 ( $m$; H-15a), 1,27 ( $m$; H-15b), 1,54 ( $m ; \mathrm{H}-16 \mathrm{a}) ; 1,36$ ( $m ; \mathrm{H}-16 \mathrm{~b}), 1,56$ ( $m$; H-18), 1,37 ( $m ; \mathrm{H}-19), 1,49$ ( $m ; \mathrm{H}-21), 1,48$ ( $m ; \mathrm{H}-22), 0,87$ ( $d ; J$ $=6,5 \mathrm{~Hz} ; \mathrm{H}-23), 0,73(s ; \mathrm{H}-24), 0,88(s ; \mathrm{H}-25), 1,01(s ; \mathrm{H}-26), 1,05$ $(s ; \mathrm{H}-27), 1,18(s ; \mathrm{H}-28), 1,00(s ; \mathrm{H}-29), 0,95$ ( $s ; \mathrm{H}-30)$. RMN de ${ }^{13} \mathrm{C}\left(125 \mathrm{MHz}, \mathrm{CDCl}_{3}, \delta\right): 22,3$ (C-1); 41,5 (C-2); 213,2 (C-3), 58,2 (C-4); 42,1 (C-5); 41,3 (C-6); 18,2 (C-7), 53,3 (C-8); 37,5 (C-9); 59, 5 (C-10); 35,6 (C-11); 30,5 (C-12); 39,7 (C-13); 38,3 (C-14); 32, 8 (C15); 36,0 (C-16); 30,0 (C-17); 42,8 (C-18); 35,4 (C-19); 28,2 (C-20); 32,4 (C-21); 39,3 (C-22); 6,8 (C-23); 14,7 (C-24); 17,9 (C-25); 20,3 (C-26); 18,7 (C-27); 32,1 (C-28); 31,8 (C-29); 35,0 (C-30).

\section{3-3-Friedelanol (4)}

Sólido branco, cristais em agulha, Tf $(243-247,8){ }^{\circ} \mathrm{C}$, não corrigido. EM (IE, $70 \mathrm{eV}$ ), $\mathrm{m} / z$ (\%): 413 (12), 275 (10), 123 (45), 55 (57), 81 (70), 69 (80) e 95 (98). RMN de ${ }^{1} \mathrm{H}\left[500 \mathrm{MHz}, \mathrm{CDCl}_{3}, \delta, J\right.$ (Hz)]: 1,43 ( $m ; \mathrm{H}-1), 1,90(d t ; J=10,0 ; 2,3 ; \mathrm{H}-2 \mathrm{a}), 1,56(m ; \mathrm{H}-2 \mathrm{~b})$, 3,73 ( $m$; H-3), 0,94 ( $s$; H-4), 1,74 ( $d t ; J=10,0 ; 3,0$; H-6a), 1,45 ( $m$; H-6b), 1,57 ( $m$; H-18), 0,91 ( $m$; H-22), 0,93 ( $d$; $J=7,3 ; \mathrm{H}-23), 0,97$ ( $s$; $\mathrm{H}-24), 0,86(s, \mathrm{H}-25), 0,99(s ; \mathrm{H}-26), 1,01$ ( $s ; \mathrm{H}-27), 1,17$ ( $s$; H-28), $0,95$ ( $s ; \mathrm{H}-29) ; 1,00(s, \mathrm{H}-30)$. RMN de ${ }^{13} \mathrm{C}\left(125 \mathrm{MHz}, \mathrm{CDCl}_{3}, \delta\right)$ : 15,8 (C-1); 36,3 (C-2); 72,9 (C-3); 49,4 (C-4); 38, 1 (C-5); 41,9 (C-6); 17,6 (C-7); 53,4 (C-8); 37,4 (C-9); 61,6 (C-10); 35,6 (C-11); 30,6 (C-12); 38,6 (C-13); 39,9 (C-14); 32,3 (C-15); 35,8 (C-16); 30,0 (C17); 43,1 (C-18); 35,4 (C-19); 28, 2 (C-20); 32,8 (C-21); 39,5 (C-22); 11,6 (C-23); 16,4 (C-24); 18,2 (C-25); 20,1 (C-26); 18,6 (C-27); 32,1 (C-28); 35,2 (C-29); 31,8, (C-30). 
Tabela 2. Dados de RMN (400 MHz, $\left.\mathrm{CDCl}_{3}\right)$ dos epímeros $(6 S, 9 R)$ e $(6 R, 9 R)$-blumenol B

\begin{tabular}{|c|c|c|c|c|c|c|}
\hline \multirow[b]{2}{*}{ Num. } & \multicolumn{3}{|c|}{ 6S,9R-blumenol B (1) } & \multicolumn{3}{|c|}{ 6R,9R-blumenol B (2) } \\
\hline & ${ }^{13} \mathrm{C}$ & $\begin{array}{l}{ }^{1} \mathrm{H} \text { mult. } \\
(J \text { em Hz })\end{array}$ & $\mathrm{HMBC}^{\mathrm{a}}$ & ${ }^{13} \mathrm{C}$ & $\begin{array}{l}{ }^{1} \mathrm{H} \text { mult. } \\
(J \text { em Hz })\end{array}$ & $\mathrm{HMBC}^{\mathrm{a}}$ \\
\hline 1 & $41,7 \mathrm{qC}$ & & & 41,7 & & \\
\hline 2 & $50,02 \mathrm{CH}_{2}$ & $\begin{array}{c}2,25 d d 18,0 ; 1,2 \\
2,50 d 18,0\end{array}$ & $\begin{array}{c}1,3,4,6,12 \text { e } 13 \\
1,3,12 \text { e } 13\end{array}$ & 50,04 & $\begin{array}{c}2,23 d d 18,0 ; 1,2 \\
2,53 d 18,0\end{array}$ & $\begin{array}{c}1,3,4,6,12 \text { e } 13 \\
1,3,12 \text { e } 13\end{array}$ \\
\hline 3 & $198,0 \mathrm{qC}$ & & & 197,9 & & \\
\hline 4 & $126,1 \mathrm{CH}$ & $5,86 d q 1,4 ; 1,2$ & 2,6 e 11 & 126,0 & $5,84 d q 1,4 ; 1,2$ & 2,6 e 11 \\
\hline 5 & $168,1 \mathrm{qC}$ & & & 167,6 & & \\
\hline 6 & $77,82 \mathrm{qC}$ & & & 77,80 & & \\
\hline 7 & $34,8 \mathrm{CH}_{2}$ & $\begin{array}{l}1,84 m \\
1,99 m\end{array}$ & $\begin{array}{l}1,5,6,8 \text { e } 9 \\
1,5,6,8 \text { e } 9\end{array}$ & 33,54 & $\begin{array}{l}1,72 m \\
1,91 m\end{array}$ & $\begin{array}{l}1,5,6,8 \text { e } 9 \\
1,5,6,8 \text { e } 9\end{array}$ \\
\hline 8 & $33,3 \mathrm{CH}_{2}$ & $\begin{array}{l}1,53 m \\
1,64 m\end{array}$ & $\begin{array}{l}6,7,9 \text { e } 10 \\
6,7,9 \text { e } 10\end{array}$ & 33,50 & $\begin{array}{l}1,53 m \\
1,64 m\end{array}$ & $\begin{array}{c}6,7,9 \text { e } 10 \\
6,9 \text { e } 10\end{array}$ \\
\hline 9 & $68,8 \mathrm{CH}$ & $3,79 m$ & 7,8 e 10 & 68,8 & $3,79 m$ & 7,8 e 10 \\
\hline 10 & $24,2 \mathrm{CH}_{3}$ & $1,23 d 6,2$ & 8 e 9 & 24,2 & $1,23 d 6,2$ & 8 e 9 \\
\hline 11 & $21,7 \mathrm{CH}_{3}$ & $2,06 d 1,4$ & 4,5 e 6 & 21,0 & $2,03 d 1,4$ & 4,5 e 6 \\
\hline 12 & $23,9 \mathrm{CH}_{3}$ & $1,06 s$ & $1,2,6$ e 13 & 23,7 & $1,09 s$ & $1,2,3,6$ e 12 \\
\hline 13 & $23,8 \mathrm{CH}_{3}$ & $1,09 s$ & $1,2,3,6$ e 12 & 24,0 & $1,06 s$ & $1,2,6$ e 13 \\
\hline
\end{tabular}

Dados obtidos dos espectros de RMN de ${ }^{1} \mathrm{H}$ e ${ }^{13} \mathrm{C}$ e DEPT-135; atribuições feitas com base nas correlações ${ }^{1} \mathrm{H}-{ }^{13} \mathrm{C}$ direta e a longa distância dos experimentos

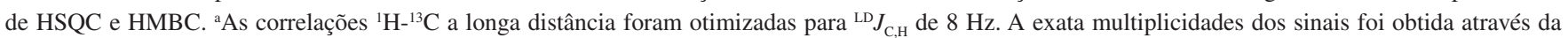
correlação $\delta$ - $J$ de experimentos de $J$ resolvido, bem como com auxílio do programa computacional first-order multiplet simulator/check FOMSC. ${ }^{14}$

Tabela 3. Atividade de extratos, frações e compostos da casca do caule de Gustavia elliptica contra formas promastigotas de L.(V) braziliensis e L.(V) guyanensis

\begin{tabular}{|c|c|c|}
\hline Amostra & $\begin{array}{c}\text { L. (V) braziliensis } \\
\mathrm{CI}_{50} / 24 \mathrm{~h}\left(\mu \mathrm{g} \mathrm{mL} \mathrm{mL}^{-1}\right)\end{array}$ & $\begin{array}{c}L(V) \text { guyanensis } \\
\mathrm{CI}_{50} / 24 \mathrm{~h}\left(\mu \mathrm{g} \mathrm{mL}^{-1}\right)\end{array}$ \\
\hline Extrato em hexano & 17,0 & NT \\
\hline Extrato em clorofórmio & 6,4 & 7,7 \\
\hline Extrato em metanol & 10,0 & NT \\
\hline Grupo C & $\mathrm{NC}$ & $\mathrm{NC}$ \\
\hline Grupo G & 197,8 & 290,6 \\
\hline Friedelina (3) & NT & $\mathrm{NC}$ \\
\hline Pentamidina* & 1,0 & 0,3 \\
\hline
\end{tabular}

*Fármaco de referência. $\mathrm{NC}=$ não calculado por dificuldade de visualização devido a grande concentração de parasitos. NT = não testado.

\section{RESULTADOS E DISCUSSÃO}

\section{Estudos fitoquímicos}

O estudo fitoquímico das folhas e cascas do caule de Gustavia elliptica resultou na obtenção de uma mistura do blumenol B e seu epimero 6-epiblumenol B (1 e 2), bem como de triterpenos (3-6, 11 e 13), sesquiterpenos (14 e 16) e ésteres de ácidos graxos (7-10 e 15). As estruturas dessas substâncias foram determinadas com base em análises de CG-EM, IV e/ou RMN 1D e 2D e comparação com dados da literatura. ${ }^{14-18}$

No espectro de IV da mistura de $\mathbf{1}$ e $\mathbf{2}$ foram observadas bandas de absorções em 3403, 1710 e $1650 \mathrm{~cm}^{-1}$, evidenciando a presença de hidroxila e carbonila $\alpha, \beta$-insaturada. Na análise por CG-EM um único pico foi registrado no cromatograma de íons totais da amostra $\left(t_{R}=23,489 \mathrm{~min}\right)$, em cujo espectro de massas foram observados os picos em $m / z, 226\left(\mathrm{M}^{+*}\right), 208\left(\mathrm{M}^{+\bullet}-\mathrm{H}_{2} \mathrm{O}\right), 152\left(\mathrm{M}^{+\bullet}-\mathrm{C}_{4} \mathrm{H}_{10} \mathrm{O}\right)$ e $110\left(\mathrm{M}^{+\bullet}-\mathrm{C}_{4} \mathrm{H}_{10} \mathrm{O}-\mathrm{C}_{2} \mathrm{H}_{2} \mathrm{O}\right)$, compatíveis com a fórmula molecular $\mathrm{C}_{13} \mathrm{H}_{22} \mathrm{O}_{3}$. Embora não distinguidos por CG-EM, os epímeros $1 \mathrm{e}$ 2 foram detectados e identificados na mistura a partir das análises dos espectros de RMN 1D e 2D, conforme será demonstrado na discussão a seguir, em que os pares de sinais de $\mathbf{1}$ e $\mathbf{2}$ distinguidos nesses espectros são mencionados separados por uma barra, sendo o primeiro sinal atribuído a $\mathbf{1}$ e o segundo a $\mathbf{2}$.

Nos espectros de RMN de ${ }^{13} \mathrm{C}\left\{{ }^{1} \mathrm{H}\right\}$ e DEPT-135 foram observados 23 sinais de carbono, 20 dos quais formam pares de sinais de mesma multiplicidade quase superpostos. Os três sinais não duplicados têm maiores intensidades relativas e foram atribuídos a sinais correspondentes de 1 e 2 com deslocamentos químicos coincidentes. Os pares de sinais em $\delta 198,0 / 197,9 ; 168,1 / 167,6$ e 126,1/126,0 confirmaram a presença dos sistemas carbonílicos $\alpha, \beta$-insaturados nos dois epímeros. Analogamente, no espectro de RMN de ${ }^{1} \mathrm{H}$ foram detectados 6 pares de sinais, cada qual coincidente nas respectivas multiplicidades e constantes de acoplamentos e com deslocamentos químicos muito próximos. Pela integração do par de sinais menos sobrepostos, em $\delta 2,06 / 2,03$, foi possível estimar a proporção relativa de 3:2 entre os epímeros 1 e 2.

Sinais de hidrogênios olefínicos dos epímeros em $\delta 5,86 / 5,84$ foram observados, no HSQC, em correlações diretas ${ }^{1} \mathrm{H}-{ }^{13} \mathrm{C}$ com os respectivos carbonos olefínicos em $\delta 126,1 / 126,0$ e, no HMBC, em correlações a longa distância ${ }^{1} \mathrm{H}-{ }^{13} \mathrm{C}$ com sinais de carbonos oxigenados em $\delta 77,82 / 77,80$, metilênicos em $\delta$ 50,02/50,04 e metílicos em $\delta 21,7 / 21,0$. Coerentemente, esses sinais de hidrogênios olefínicos, ambos duplos quartetos $(J=1,4$ e 1,2 Hz), foram observados no COSY em correlações ${ }^{1} \mathrm{H}-{ }^{1} \mathrm{H}$ com hidrogênios metilênicos em $\delta$ 2,25/2,23 e de metila ligada a carbono $\mathrm{sp}^{2}$ em $\delta 2,06 / 2,03$. Estes hidrogênios, ligados aos carbonos metílicos em $\delta 21,7 / 21,0$ (HSQC), têm correlações com os carbonos em $\delta 168,1 / 167,6 ; \delta 126,1 / 126,0$ e $\delta 77,82 / 77,80$ (HMBC). Por sua vez, os hidrogênios metilênicos em $\delta 2,25 / 2,23$, identificados como axiais e os respectivos hidrogênios equatoriais em 
$\delta 2,50 / 2,53$ estão conectados aos carbonos em $\delta 50,02 / 50,04$, (HSQC), e têm correlações a longa distância ${ }^{1} \mathrm{H}-{ }^{13} \mathrm{C}$ (HMBC) com os carbonos carbonílicos em $\delta 198,0 / 197,9$ e com carbonos não hidrogenados em $\delta 41,7$ e metílicos em $\delta$ 23,9/24,0 e 23,8/23,7. Para os hidrogênios axiais foram ainda observadas correlações com os carbonos em $\delta$ $77,82 / 77,80$ e $\delta 126,1 / 126,0$. Estes dados são compatíveis com a parte anelar e respectivas metilas de $\mathbf{1}$ e $\mathbf{2}$.

A cadeia lateral, evidenciada no espectro de massas pelo íon de $\mathrm{m} / \mathrm{z}, 152\left(\mathrm{M}^{+\bullet}-\mathrm{C}_{4} \mathrm{H}_{10} \mathrm{O}\right)$ foi confirmada pelos sinais de carbonos metilênicos em $\delta 34,8 / 33,54$ e 33,3/33,50, carbinólico metínico em $\delta 68,8$ e metílico em $\delta 24,2$ nos espectros de RMN de ${ }^{13} \mathrm{C}\left\{{ }^{1} \mathrm{H}\right\}$ e DEPT-135, bem como pelas correlações ${ }^{1} \mathrm{H}-{ }^{13} \mathrm{C}$ direta e a longa distância nos experimentos de HSQC e HMBC. Estes e os demais dados (Tabela 2) confirmaram a presença de $\mathbf{1}$ e $\mathbf{2}$ na amostra.

A identificação de $\mathbf{1}$ com o blumenol B e, consequentemente, sua configuração absoluta foi estabelecida pela comparação com dados publicados. ${ }^{15,18}$ Para atribuir a configuração absoluta de $\mathbf{2}$ levou-se em consideração que todos os sinais duplicados nos espectros de RMN de ${ }^{1} \mathrm{He}{ }^{13} \mathrm{C}\left\{{ }^{1} \mathrm{H}\right\}$ dos dois compostos (Tabela 2) pertencem ou estão ligados à parte anelar da estrutura, indicando que a inversão de configuração ocorre no carbono estereogênico C-6 da junção da cadeia lateral. Claramente os sinais de $\mathrm{RMN}$ de ${ }^{1} \mathrm{He}{ }^{13} \mathrm{C}$ da parte terminal da cadeia lateral, C-9 e C-10 não aparecem duplicados, ratificando que a epimeria de 2 em relação ao blumenol B (1) não deve ser em C-9. Para efeitos comparativos, considerou-se o blumenol A glicosilado e seu epímero em C-9, ${ }^{16}$ cujos respectivos dados apresentam diferenças apenas nos carbonos da cadeia lateral (C-7 a C-10). Se para tais compostos a inversão de configuração em C-9 não causa distinção dos sinais de RMN dos átomos anelares, mesmo sendo relativamente volumoso o grupo a ele ligado, as diferenças observadas entre $\mathbf{1}$ e $\mathbf{2}$ só podem ter sido causadas pela inversão em C-6. Portanto, tendo sido observada a identidade de $\mathbf{1}$ com o $(6 S, 9 R)$-blumenol $\mathrm{B},{ }^{15,18}$ sugere-se que 2 seja o $(6 R, 9 R)$-blumenol B ou 6-epiblumenol B, o qual está sendo descrito primeira vez como produto natural.

Norisoprenoides com 9 a 13 átomos de carbonos são compostos flavorizantes cuja origem é associada à degradação de carotenoides, tendo sido identificados no tabaco e em frutos como marmelo, manga e, principalmente, em vinhos e sucos de uva. ${ }^{19-21}$ Embora tais compostos tenham sido encontrados em espécies de outras famílias, como Apocynaceae, Euphorbiaceae, Anacardiaceae, Boraginaceae e Rubiaceae, ${ }^{22}$ esta é a primeira descrição da presença dos epímeros do blumenol B na família Lecythidaceae, evidenciando sua ampla ocorrência. Com relação à importância biológica do blumenol B foram descritos resultados de atividades alelopática. ${ }^{23}$

Além dos norisoprenoides $\mathbf{1}$ e $\mathbf{2}$, o estudo fitoquímico das cascas do caule e das folhas de G. elliptica resultou na obtenção dos triterpenos 3-6 e 13-17, ésteres graxos 7-10 e 15 e sesquiterpenos 14 e 16 (Tabela 1). Entre os triterpenos isolados destacam-se a friedelina (3), como composto majoritário encontrado nas duas partes da planta, e o friedelanol (4), encontrado em menor proporção, o qual apresenta atividade anti-inflamatória. ${ }^{24}$ Entre os sesquiterpenos destacam-se o trans-cariofileno (14), com atividade antimicrobiana, e o $\alpha$-humuleno (16), com atividades inseticida, antimicrobiana, antioxidante e anticancerígena, ${ }^{25,26}$ enquanto que entre os ésteres graxos destaca-se o hidnocarpato de etila (15), um dos primeiros medicamentos usados no tratamento da lepra. ${ }^{27}$

\section{Ensaios biológicos}

De todos os extratos das cascas do caule de G. elliptica avaliados, o extrato em clorofórmio foi o que apresentou maior percentagem de inibição para as cepas de Leishmania (V) braziliensis e L. (V) guyanensis, enquanto que os extratos em hexano e metanol apresentaram alta percentagem de inibição somente contra $L$. (V) braziliensis. Os grupos $\mathrm{C}$ e $\mathrm{G}$ (Tabela 1), selecionados para ensaio, por apresentarem constituintes majoritários ou registro de atividades biológicas comprovadas $^{24-26}$ foram fracamente ativos ou inativos (Tabela 3). Por outro lado, o triterpeno friedelina (3), além de inativo, aparentemente, estimulou a proliferação dos parasitos.

\section{CONCLUSÃO}

O estudo fitoquímico da espécie Gustavia elliptica confirmou a composição rica em terpenos e ácidos graxos característica do gênero, sendo a friedelina (3) o constituinte majoritário nas duas partes investigadas da planta. A presença de norisoprenoides na família Lecythidaceae é descrita pela primeira vez, sendo identificados os epímeros $(6 S, 9 R)$ e $(6 R, 9 R)$-blumenol B (1 e 2, respectivamente), este inédito como produto natural. As configurações absolutas sugeridas para 1 e 2 tiveram por base dados da literatura. ${ }^{15,18}$ Os resultados dos ensaios antileishmania, com os extratos da planta são promissores e justificam o uso popular de espécies de Gustavia no combate à leishmaniose, estimulando a continuação das investigações fitoquímicas deste gênero.

\section{MATERIAL SUPLEMENTAR}

Os espectros de RMN de ${ }^{1} \mathrm{H}$ e ${ }^{13} \mathrm{C}$ da mistura de $\mathbf{1}$ e 2 estão disponíveis em http://quimicanova.sbq.org.br na forma de arquivo PDF, com acesso gratuito.

\section{AGRADECIMENTOS}

À CAPES, ao CNPq, à FAPEAM e FINEP pelo suporte financeiro e bolsas de estudos

\section{REFERÊNCIAS}

1. Prance, G. T.; Mori, S. A.; Lecythidaceae- Parte I: The actinomorphicflowered New World Lecythidaceae (Asteranthos, Gustavia, Grias, Allantoma \& Cariniana). Flora Neotropica Monograph 21, New York Bot. Gard: New York, 1979, p. 270.

2. Grenand, P.; Moretti, C.; Jacquemin, H.; Pharmacopées traditionnelles en Guyane - Créole, Palikur, Wayãpi, I'Orstom: Paris, 1987.

3. Souza, A. D. L de; Rocha, A. F. I. da; Pinheiro, M. L. B.; Andrade, C. H. de S.; Galotta, A. L. de A. Q.; Santos, M. de P. S. S. dos S.; Quim. Nova 2001, 24, 439.

4. El-Seedi, H. R.; Zayed, M. F.; Morono, F. G.; Torssell, K. B. G.; Rev. Latin. Quim. 1999, 27, 56.

5. Pettir, G. R.; Zhang, Q.; Pinilla, V.; Herald, D. L.; Douber, D. L.; Duke, J. A.; J. Nat. Prod. 2004, 67, 983.

6. Campos, F. R.; Januário, A. H.; Rosas, L. V.; Nascimento, S. K. R.; Pereira, P. S.; França, S. C.; Cordeiro, M. S. C.; Toldo, M. P. A.; Albuquerque, S.; Fitoterapia 2005, 76, 26.

7. Bastos, D. Z. L.; Pimentel, I. C.; Jesus, D. A. de; Oliveira, B. H. de; Phytochemistry 2007, 68, 834.

8. Ribeiro, J. E. L. da S.; Hopkins, M. J. G.; Vicentini, A.; Sothers, C. A.; Costa, M. A. da S.; Brito, J. M. de; Souza, M. A. D. de; Martins, L. H. P.; Lohmann, L. G.; Assunção, P. A. C. L.; Perreira, E. da C.; Silva, C. F.; Mesquita, M. R.; Procópio, L. C.; Flora da Reserva Ducke: Guia de Identificação das plantas vasculares de uma floresta de terra-firme na Amazônia Central, INPA: Manaus, 1999

9. Adams, R. P.; Identification of Essential Oil Components by Gas Chromatography/Quadrupole Mass Spectroscopy, Allured Publ.: Carol Stream, 2001.

10. Novy, F. G.; Macneal, W. J.; J. Infect. Dis. 1904, $1,1$. 
11. Nicolle, G. L; Acad. Sci. Paris 1908, 146, 842.

12. Hendricks, L.; Wright, N.; Am. J. Trop. Med. 1979, 28, 962.

13. Canto-Cavalheiro, M. M.; Echevarria, A.; Araujo, C. A.; Bravo, M. F.; Santos, L. H.; Jansen, A. M.; Leon, L. L.; Micróbios 1997, 90, 51.

14. http://artemis.ffclrp.usp.br/NMR.htm, acessada em Março 2011.

15. Erosa-Rejón, G.; Peña-Rodríguez, L. M.; Sterner, O.; J. Mex. Chem. Soc. 2009, 53, 44.

16. Correia, S. J.; David, J. M.; Silva, E. P.; David, J. P.; Lopes, L. M. X.; Guedes, M. L. S.; Quim. Nova 2008, 31, 2056.

17. Marino, S. De; Gala, F.; Zollo, F.; Vitalini, S.; Fico, G.; Visioli, F.; Iorizzi, M.; Molecules 2008, 13, 1219.

18. Weiss, G.; Koreeda, M.; Nakanishi, K.; J.C.S. Chem. Commun. 1973, 565 .

19. Winterhalter, P.; Schreier, P.; J. Agric. Food Chem. 1988, 36, 1251.

20. Skouroumounis, G. K.; Winterhalter, P.; J. Agric. Food Chem. 1994, 42, 1068.
21. Ollé, D.; Baumes, R. L.; Bayonove, C. L.; Lozano, Y. F.; Sznaper, C.; Brillouet, J. M.; J. Agric. Food Chem. 1998, 46, 1094.

22. Stuart, K. L.; Woo-Ming, R.; Phytochemistry 1975, 14, 594.

23. Tseng, M.; Kuo, Y.; Chen, Y.; Chou, C.; J. Chem. Ecol. 2003, 29, 1269.

24. Supudompol, B.; Wongseripipatana, S.; Likhitwitayawuid, K.; J. Sci. Technol. 2005, 27, 563.

25. Almeida, L. F. R.; Delachiave, M. E. A.; Marques, M. O. M.; Rev. Bras. Pl. Med. 2005, 8, 35 .

26. Fernandes, E. S.; Passos, G. F.; Medeiros, R.; Cunha, F. M.; Ferreira, J.; Campos, M. M.; Pianowski, L. F.; Calixto, J. B.;Eur. J. Pharmacol. 2007, 569, 228.

27. Oliveira, A. S.; Lima, J. A.; Rezende, C. M.; Pinto, A. C.; Quim. Nova 2009, 32, 139 . 
Maria de Fátima Oliveira Almeida, Ana Cláudia Rodrigues de Melo, Maria Lúcia Belém Pinheiro, Jefferson Rocha de Andrade Silva e Afonso Duarte Leão de Souza*

Departamento de Química, Universidade Federal do Amazonas, Av. General Rodrigo Octávio Jordão Ramos, 3000, Campus Universitário, setor Sul, 69077-000 Manaus - AM, Brasil

Andersson Barison e Francinete Ramos Campos

Departamento de Química, Universidade Federal do Paraná, Centro Politécnico, CP 19081, 81531-990 Curitiba - PR, Brasil Ana Cláudia Fernandes Amaral

Instituto de Tecnologia de Fármacos, Farmanguinhos, Fundação Oswaldo Cruz, Av. Brasil, 4365, 21041-250, Rio de Janeiro - RJ, Brasil

Gerzia Maria de Carvalho Machado e Leonor Laura Pinto Leon

Departamento de Imunologia, Fundação Oswaldo Cruz, Av. Brasil, 4365, 21045-900 Rio de Janeiro - RJ, Brasil

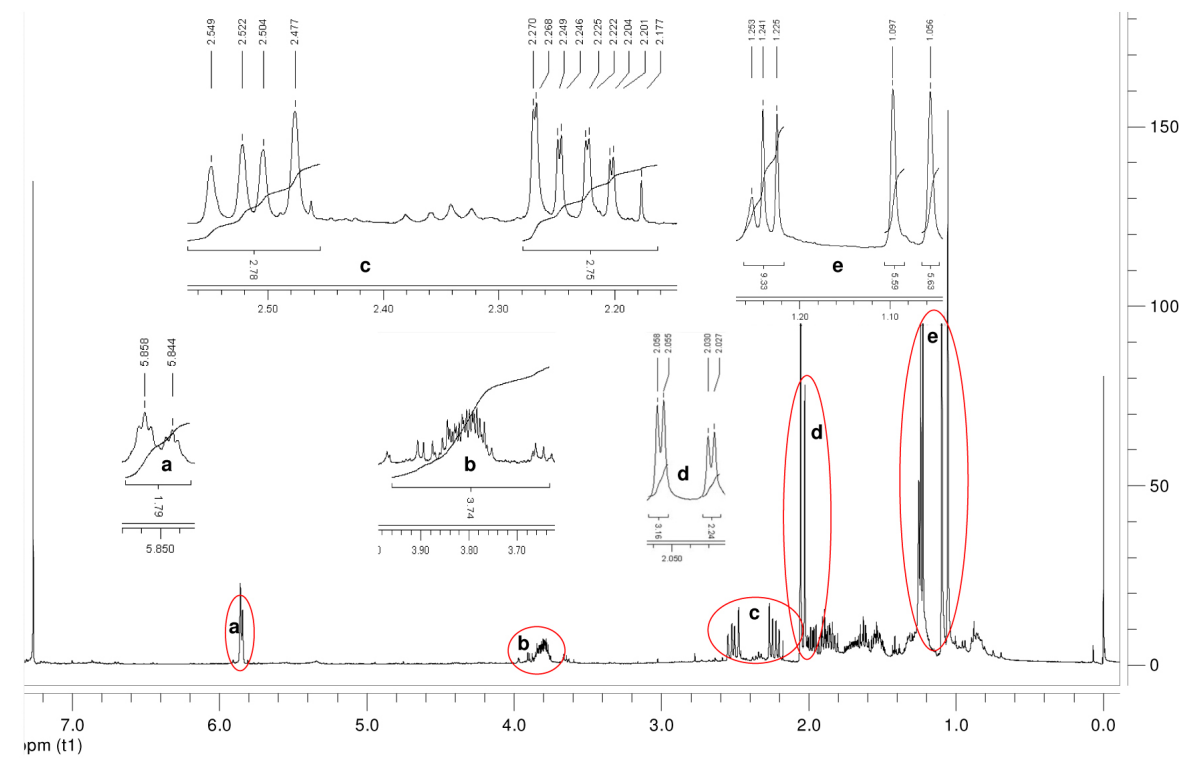

Figura 1S. Espectro de $\mathrm{RMN}$ de ${ }^{1} \mathrm{H}$ de 1 e $2\left(400 \mathrm{MHz}, \mathrm{CDCl}_{3}\right)$ com expansões parciais
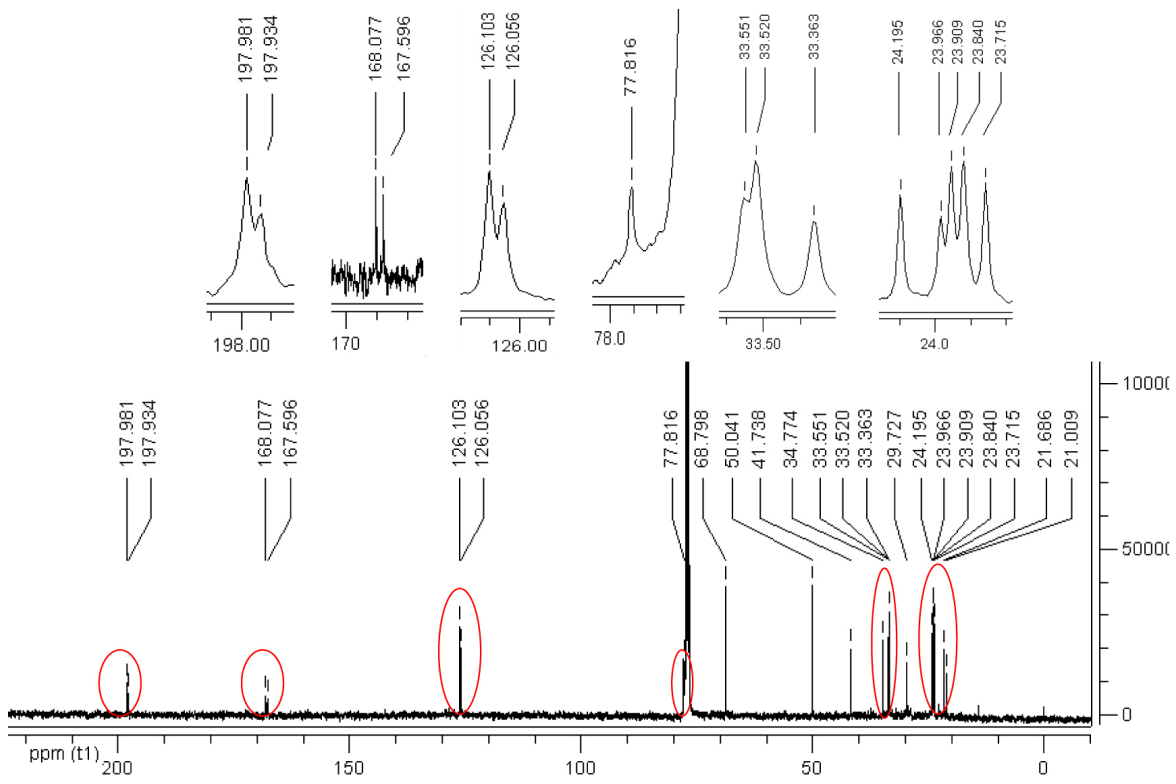

Figura 2S. Espectro de $\mathrm{RMN}$ de ${ }^{13} \mathrm{C}$ de 1 e $2\left(100 \mathrm{MHz}, \mathrm{CDCl}_{3}\right)$ com expansões parciais

*e-mail: souzadq@ufam.edu.br 